\section{P-263 NURSES SPECIAL INTEREST PROJECT-ENCOURAGING INNOVATION, INCREASING STAFF INVOLVEMENT, RECOGNISING ACHIEVEMENT}

Gemma Hodge. St Gemma's Hospice, Leeds, West Yorkshire

\subsection{6/bmjspcare-2016-001245.282}

The hospice recognised the need to maximise the talent and expertise of Band 5 palliative care nurses. Funding was set aside in annual budget planning to reward the equivalent of one increment on Agenda for Change NHS pay scale (band 5) to nurses undertaking a project for one year. We later diversified the plan to also allow band 3 healthcare assistants to be eligible which again rewards the equivalent of one increment on Agenda for Change NHS pay scale (band 3 ).

Through setting up a project, nurses could be recognised for the additional work they do, over and above caring for the patients. As a hospice we know we are providing the best evidence based care, whilst in turn upskilling staff.

Staff create a project plan with a number of outcomes, often including increasing staff awareness of a current evidence on their topic and creating resources for staff to use.

We support staff by offering dedicated study time, access to study days and ongoing project support from our advanced nurse practitioner. In return they deliver current evidence based resources and teaching to the in patient unit staff.

On launch of project uptake was low with three projects being submitted, once these projects were in action and visible on the wards, we opened a second round of application, to utilise the funding allocated. There was increased interest, generating many applications from staff. This highlights staff clearly value the project and are keen to get involved.

We currently have five projects running covering topics including, Motor Neurone Disease, Wound care, Outcome Measures and Infection Prevention. We are planning poster displays to celebrate nursing achievements in the hospice to highlight the work being undertaken and how this is influencing our practice.

\section{P-264 GROW YOUR OWN PALLIATIVE CARE CLINICAL NURSE SPECIALISTS}

Claire Blakey, Christine Barnes. Ashgate Hospice, Chesterfield, UK

\subsection{6/bmjspcare-2016-001245.283}

Background A National Council for Palliative Care workforce survey (2011) highlighted a predicted specialist nurse workforce crisis with the ageing workforce and difficulties in recruiting the required levels of skill and competence.

Estimates show $25-65 \%$ of the population will require specialist palliative care in the last year of life. Consequently, the PCCNS workload is predicted to increase significantly and planning is required to meet this demand (Calanzani et al., 2013).

Locally, there is an inability to recruit PCCNS with the required skills, knowledge and experience, this is reflected nationally (Macmillan Cancer Support, 2014). Innovative ways to develop the workforce include introducing palliative care development posts, this was regarded as an opportunity to introduce skill mix whilst developing the specialist nurses of the future.

Introducing development posts has enabled comprehensive mentorship from expertise within the existing team with the ethos to 'grow your own' and develop the Hospice's PCCNS workforce of the future.
A comprehensive development framework programme has been produced for completion by all palliative care nurse development post-holders over a two year period gaining experience within both the acute hospital and community settings.

Aim Implementation of the development programme is to ensure the organisation has a future workforce of PCCNS with the required skills, knowledge, values and expertise.

Method Completion of a Development Framework, encompassing clinical expertise, leadership, innovation and education.

Potential Outcomes: Standardised, evidence based and individualised approach to the development of future PCCNS across acute and community services.

Clinical and experiential skills within the existing team are 'passed on' to the future workforce encompassing the values, attitudes and behaviours associated with the PCCNS role.

Current and Future Relevance

Pro active and innovative way of responding to workforce crisis.

Promoting the ethos of the organisation.

Recognising and supporting achievement and individual ambition.

Potential contribution nationally to the development of PCCNS.

\section{P-265 PALLIATIVE CARE CLINICAL NURSE SPECIALIST: A NEW ADDITION TO THE HOSPICE INPATIENT UNIT TEAM}

Lily Chan, Simon Walker, Andrew Fletcher. St Catherine's Hospice, Preston, UK

\subsection{6/bmjspcare-2016-001245.284}

Background In December 2014 the hospice introduced the role of CNS on to the inpatient unit. The role involves working closely with the medical team in admission and management of patients whilst providing mentorship and education to nursing staff and junior doctors.

Aim To determine the effectiveness and impact of the CNS role on the hospice inpatient unit.

Methods An online anonymous survey of the multidisciplinary inpatient team.

Results 18 members of the multidisciplinary team responded. $28 \%$ of staff understood the CNS role very well with $72 \%$ moderately well. All staff found the addition of the CNS role to be useful with $78 \%$ feeling the role was very or extremely useful. $72 \%$ and $67 \%$ of staff found the role to be of great value to patients and their families respectively whilst $71 \%$ felt one of the most valuable aspects of the role was being a link between the medical and non-medical teams. Comments highlighted the nursing experience and clinical knowledge of the CNS to be of particular benefit. Having CNS input within the medical team offers a different perspective on case management.

Discussion The survey results and experience of the team support the conclusion that the introduction of the CNS role on the hospice inpatient unit has been highly effective for patients, their families and the multidisciplinary team. The CNS had completed a clinical assessment and diagnostics qualification prior to commencing the role which proved to be of great value and would be seen as a very desirable qualification in the future. Since commencing the role, the CNS is now a non-medical prescriber which is proving to be of significant benefit. Later in the year, training for the non-medical prescribing of blood components will be completed. A survey of patients and families views is also to be undertaken. 